DOI: 10.5604/01.3001.0012.0700

\title{
MAGNETIC FIELD OF COAXIAL SQUARE COILS ENCLOSED WITH HIGH-PERMEABILITY MATERIAL
}

\author{
Anamarija Juhas ${ }^{1}$, Neda Pekaric Nad ${ }^{1}$, Hannes H. Toepfer ${ }^{2}$ \\ ${ }^{1}$ University of Novi Sad, Faculty of Technical Science/Department of Power, Electronic and Telecommunication Engineering, ${ }^{2}$ Technische Universität Ilmenau, Department \\ of Advanced Electromagnetics
}

Abstract. In this paper, the method of images is used to model the effects of enclosure made of high-permeability material on magnetic field of square coils. Closed-form description for the images, along with the case study involving square Helmholtz coils, is provided.

Keywords: Helmholtz coils, magnetic field, method of images

\section{POLE MAGNETYCZNE WSPÓLOSIOWYCH KWADRATOWYCH CEWEK ZAMKNIĘTYCH W MATERIALE O WYSOKIEJ PERMEANCJI}

Streszczenie. W artykule opisano wykorzystanie metody obrazów do modelowania wplywu obudowy wykonanej z materiału o dużej przenikalności magnetycznej w obszarze kwadratowych cewek. Przedstawiono opis obrazów dla zamkniętej formy wraz ze studium przypadku z kwadratowymi cewkami Helmholtza.

Slowa kluczowe: cewki Helmholtza, pole magnetyczne, metoda obrazów

\section{Introduction}

This paper is devoted to the analysis of magnetic field of square loops enclosed with high-permeability material. Systems like this can be found inside shielded exposure equipment for biological experiments [21], inside passive shielded rooms with MRI equipment [13], or inside shielded electronic equipment to protect it from external fields [1]. As shown in [21], the $\mu$-metal box can be used to enlarge the volume of uniform magnetic field inside the system of four coaxial square coils.

The paper is organized as follows. In Section 1 we provide formulation of the problem, describe our model as well as magnetic field calculation which is based on the method of current images. In Section 2, we extend our considerations to the system of two coaxial loops in the presence of magnetic enclosure. In this section we consider Helmholtz coils as an important special case. In Section 3, we provide the concluding remarks and some possible extensions of the presented results. The expressions used in the calculations of the magnetic flux density vector of the original and all image loops are provided in Appendix.

\section{Formulation of the problem}

Here we consider the effect of enclosure made of highpermeability material on the magnetic field of the square loop. The geometry of the problem is presented in Fig. 1. We assume that the loop is made of a conductor of zero radius. In order to simplify the analysis, we further assume that the walls of the enclosure are infinitely thick. As shown in [18], the effects of finite thickness of the walls of magnetic materials is more pronounced if the relative permeability is less than 100 (e.g. for some soft ferrites).

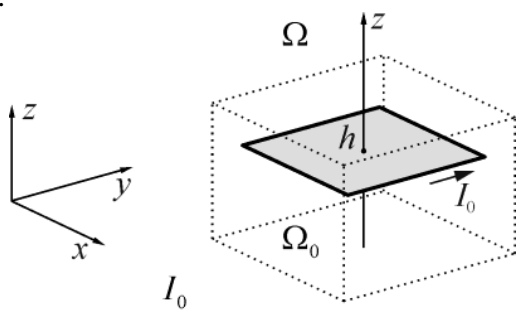

Fig. 1. Geometry of the square loop enclosed with high-permeability material

A square loop of side dimensions $2 a$ surrounded by the air (region $\Omega_{0}$ ) is enclosed with high-permeability material (region $\Omega$ ) occupying $|x| \geq d / 2,|y| \geq d / 2,|z| \geq d / 2$. To be more precise, region $\Omega_{0}$ is hole inside the infinite block of highpermeability material. The shape of the hole is the cube with side dimensions equal to $d$ and center at the origin. The coordinates of the center of the loop are $(0,0, h)$ where $|h|<d / 2$. The sides of the loop are either parallel or orthogonal to the sides of the cube. In our model, the permeability of the magnetic material is constant and equal to $\mu$. Conductivity of both media is equal to zero.

In order to take into account the influence of the enclosure on the magnetic field, the method of current images is used. It is well known that the method of images is powerful tool when the geometry of boundaries is simple. According to the method of images, magnetic flux density can be calculated by introducing the image loops (see e.g. $[11,22])$. The positions and the currents of the image loops ensure proper boundary conditions at all interfaces between the two media. The method of images enables calculation of the magnetic field in all regions except inside the conductor of the loop.

According to the method of images, the magnetic field in region $\Omega_{0}$ can be determined by removing magnetic material and introducing an infinite set of the image loops. In what follows, the $n$th layer of images $(n \geq 1)$ refers to the set of all images which are located between two concentric cubes with centers at the origin and side dimensions $(2 n-1) d$ and $(2 n+1) d$. Accordingly, the images in the first layer are located between the cubes of side dimensions $d$ and $3 d$, the images in the second layer are located between the cubes of side dimensions $3 d$ and $5 d$, etc. The number of the images in the $n$th layer is equal to $(2 n+1)^{3}-(2 n-1)^{3}=24 n^{2}+2$ (corresponding to 26 images in the first layer, 98 in the second, 218 in the third, etc.). Let us denote

$$
\alpha=\frac{\mu-\mu_{0}}{\mu+\mu_{0}} .
$$

The centers and the currents of the image loops in the $n$th layer can be obtained as

$$
\begin{gathered}
C\left(n_{x}, n_{y}, n_{z}\right)=\left(n_{x} d, n_{y} d, n_{z} d+(-1)^{n_{z}} h\right), \\
I\left(n_{x}, n_{y}, n_{z}\right)=(-\alpha)^{\left|n_{x}\right|+\left|n_{y}\right|} \cdot \alpha^{\left|n_{z}\right|} I_{0},
\end{gathered}
$$

respectively, where $\alpha$ is given by (1), $I_{0}$ denotes the current in the original loop, and $n_{x}, n_{y}$ and $n_{z}$ are integers satisfying

$$
\max \left(\left|n_{x}\right|,\left|n_{y}\right|,\left|n_{z}\right|\right)=n \text {. }
$$

The positive value of the current in the image loop means that its direction is the same as in the original loop. Contrary, the negative value corresponds to the current in opposite direction. The axis of each image loop is parallel to the axis of the original loop. According to (2)-(3), 3-tuple $\left(n_{x}, n_{y}, n_{z}\right)$ completely describes the image. The order of the layer to which the image loop belongs can be determined from (4). Moreover, the centers of all image loops 
in the $n$th layer can be generated by using the following procedure

$$
\begin{aligned}
& \text { i) } n_{x}= \pm n, \quad-n \leq n_{y} \leq n, \quad-n \leq n_{z} \leq n, \\
& \text { ii) }-n<n_{x}<n, \quad n_{y}= \pm n, \quad-n \leq n_{z} \leq n, \\
& \text { iii) }-n<n_{x}<n, \quad-n<n_{y}<n, \quad n_{z}= \pm n .
\end{aligned}
$$

Let us consider the first layer $(n=1)$ of the images. For $n=1$, first step of the above procedure reads: $n_{x}= \pm 1$, $-1 \leq n_{y} \leq 1, \quad-1 \leq n_{z} \leq 1$. This step generates 18 images listed as images $1-18$ in Table 1 . The second step $-1<n_{x}<1, n_{y}= \pm 1$, $-1 \leq n_{z} \leq 1$, generates 6 images listed as images 19-24 in Table 1 . Finally, the third step $-1<n_{x}<1,-1<n_{y}<1, \quad n_{z}= \pm 1$, generates the last two images in Table 1 . The coordinates of the center of each image loop are calculated from (2). The current in each

\begin{tabular}{|c|c|c|c|c|c|}
\hline Image no. & $n_{x}$ & $n_{y}$ & $n_{z}$ & $C\left(n_{x}, n_{y}, n_{z}\right)$ & $I\left(n_{x}, n_{y}, n_{z}\right)$ \\
\hline 1 & -1 & -1 & -1 & $(-d,-d,-d-h)$ & $\alpha^{3} I_{0}$ \\
\hline 2 & -1 & 0 & -1 & $(-d, 0,-d-h)$ & $-\alpha^{2} I_{0}$ \\
\hline 3 & -1 & 1 & -1 & $(-d, d,-d-h)$ & $\alpha^{3} I_{0}$ \\
\hline 4 & -1 & -1 & 0 & $(-d,-d, h)$ & $\alpha^{2} I_{0}$ \\
\hline 5 & -1 & 0 & 0 & $(-d, 0, h)$ & $-\alpha I_{0}$ \\
\hline 6 & -1 & 1 & 0 & $(-d, d, h)$ & $\alpha^{2} I_{0}$ \\
\hline 7 & -1 & -1 & 1 & $(-d,-d, d-h)$ & $\alpha^{3} I_{0}$ \\
\hline 8 & -1 & 0 & 1 & $(-d, 0, d-h)$ & $-\alpha^{2} I_{0}$ \\
\hline 9 & -1 & 1 & 1 & $(-d, d, d-h)$ & $\alpha^{3} I_{0}$ \\
\hline 10 & 1 & -1 & -1 & $(d,-d,-d-h)$ & $\alpha^{3} I_{0}$ \\
\hline 11 & 1 & 0 & -1 & $(d, 0,-d-h)$ & $-\alpha^{2} I_{0}$ \\
\hline 12 & 1 & 1 & -1 & $(d, d,-d-h)$ & $\alpha^{3} I_{0}$ \\
\hline 13 & 1 & -1 & 0 & $(d,-d, h)$ & $\alpha^{2} I_{0}$ \\
\hline 14 & 1 & 0 & 0 & $(d, 0, h)$ & $-\alpha I_{0}$ \\
\hline 15 & 1 & 1 & 0 & $(d, d, h)$ & $\alpha^{2} I_{0}$ \\
\hline 16 & 1 & -1 & 1 & $(d,-d, d-h)$ & $\alpha^{3} I_{0}$ \\
\hline 17 & 1 & 0 & 1 & $(d, 0, d-h)$ & $-\alpha^{2} I_{0}$ \\
\hline 18 & 1 & 1 & 1 & $(d, d, d-h)$ & $\alpha^{3} I_{0}$ \\
\hline 19 & 0 & -1 & -1 & $(0,-d,-d-h)$ & $-\alpha^{2} I_{0}$ \\
\hline 20 & 0 & 1 & -1 & $(0, d,-d-h)$ & $-\alpha^{2} I_{0}$ \\
\hline 21 & 0 & -1 & 0 & $(0,-d, h)$ & $-\alpha I_{0}$ \\
\hline 22 & 0 & 1 & 0 & $(0, d, h)$ & $-\alpha I_{0}$ \\
\hline 23 & 0 & -1 & 1 & $(0,-d, d-h)$ & $-\alpha^{2} I_{0}$ \\
\hline 24 & 0 & 1 & 1 & $(0, d, d-h)$ & $-\alpha^{2} I_{0}$ \\
\hline 25 & 0 & 0 & -1 & $(0,0,-d-h)$ & $\alpha I_{0}$ \\
\hline 26 & 0 & 0 & 1 & $(0,0, d-h)$ & $\alpha I_{0}$ \\
\hline
\end{tabular}
image loop is obtained from (3).

Table 1. The first layer of images

The positions of the centers of all image loops in the first layer are shown in Fig. 2. The numbers in the squares correspond to the numbers of the images in Table 1 . Numerical values used in this example are $d=0.5 \mathrm{~m}$ and $h=0.2 \mathrm{~m}$. The center $(0,0, h)$ of the original loop (source loop) is marked with downward triangle dot. In order to improve the clarity of the figure, the centers that belong to the planes $z=-d-h=-0.7 \mathrm{~m}, \quad z=h=0.2 \mathrm{~m}$ or $z=d-h=0.3 \mathrm{~m}$ are interconnected with black solid lines. The region $\Omega_{0}$ is region inside the cube plotted with green lines. The centers of all image loops in the first layer are located between the cubes of side dimensions $d=0.5 \mathrm{~m}$ (green lines) and $3 d=1.5 \mathrm{~m}$ (red lines).

In the following example, we compare the magnetic flux density of the square loop with and without enclosure (Fig. 3a).
Moreover, we compare the calculated values of the magnetic flux density of the enclosed square loop by taking into account only the first (Fig. 3b), and both the first and the second layer of the images (Fig. 3c). The magnetic flux density is calculated in the region $\Omega_{0}$ at the plane $y=0$. The expressions used in the calculations of the magnetic flux density of the original and image loops are provided in the Appendix. Resultant magnetic flux density in the region $\Omega_{0}$ is obtained by superposition of the fields of the original loop and the image loops. Numerical values used in the calculations are $I_{0}=1 \mathrm{~A}, \quad d=0.5 \mathrm{~m}, \quad a=0.2 \mathrm{~m}, \quad h=0.2 \mathrm{~m}$ and $\alpha=1$ (corresponding to $\left.\mu / \mu_{0} \rightarrow \infty\right)$. Since the air is linear medium, and currents in all image loops are linearly proportional to the current in the original loop (see (3)), it follows that resultant magnetic flux density is also linearly proportional to the current in the original loop. Therefore, the presented results can be easily scaled up or down for arbitrary current.

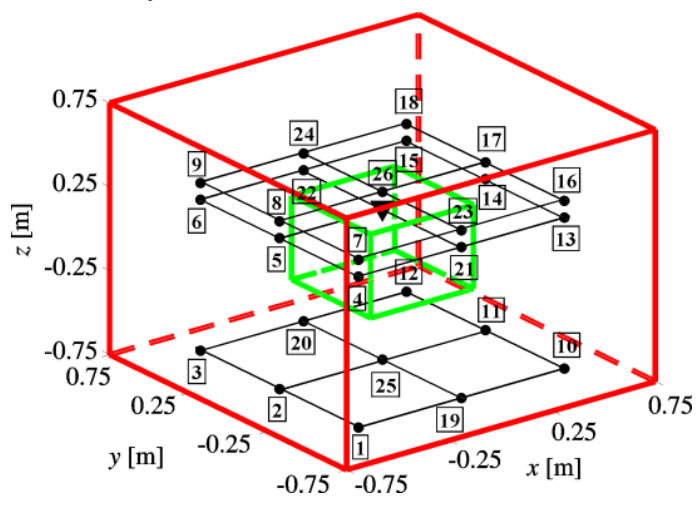

Fig. 2. The centers of all image loops in the first layer (black dots) and the center of the original loop (downward triangle dot)

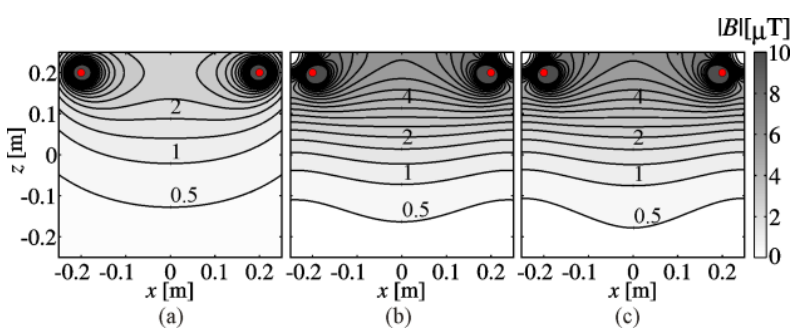

Fig. 3. Magnetic flux density of a square loop at the plane $y=0$ (a) without enclosure, (b) with enclosure by taking into account only the first layer of images and (c) with enclosure by taking into account both the first and the second layer of images

According to the definition of the layer of images, the distance between the original loop and the layer increases with increasing order of the layer. Moreover, the number of images in the layer also increases with the order of the layer. Thus, it is not obvious that the magnetic field of the higher order layers is significantly weaker than the magnetic field of lower order layers. However, comparing Figs. $3 \mathrm{~b}$ and $3 \mathrm{c}$ it follows that the effect of the second layer of images on the magnetic flux density is very weak. We also investigated the impact of the layers up to sixth order. Our numerical experiments showed that higher order layers $(3 \leq n \leq 6)$ can be completely neglected in the magnetic flux density calculations, because they produce very weak field in comparison to the field of the original loop. We also conclude that in calculations only the first layer of images should be included. We arrived to the same conclusion for the other values of $\alpha \geq 0.5$.

\section{Case study: system of two coaxial square loops}

The exposure systems for bio-magnetic experiments in in vitro studies are usually based on systems of coaxial coils connected in series and enclosed with high-conductivity and/or highpermeability material (see e.g. $[14,21])$. The enclosure represents either commercial incubator or it serves as a shield from the background fields. 
In this section we extend our analysis to the system of two loops enclosed with high-permeability material. The loops are assumed to be identical, coaxial and parallel. As an important special case we consider square Helmholtz coils. We investigate the effect of the enclosure and variation of the length of the sides of the coil (relative to the length of the edges of the enclosure) on magnetic field and its uniformity.

The geometry of two identical square loops having common axis and enclosed with high-permeability material is presented in Fig. 4. The coils are located at planes $z=h$ and $z=-h$. All other data are the same as in the previous section (Fig. 1).

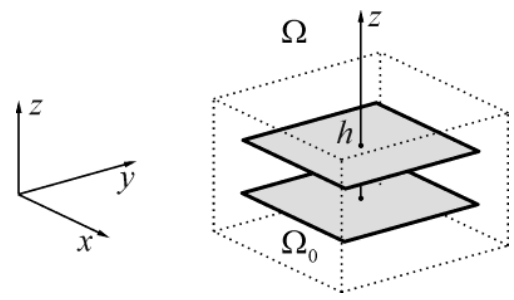

Fig. 4. System of two coaxial and parallel square loops enclosed with high-permeability material

\subsection{Helmholtz coils with enclosure}

Helmholtz coils are widely used to generate uniform magnetic field needed for bio-magnetic experiments (e.g. [14]), for the testing of equipment (e.g. $[16,23])$, as well as for calibration of magnetic field sensors (e.g. [5, 10]). In all mentioned applications, it is of significance to obtain as large as possible volume of the uniform field.

General analysis of the magnetic field in Helmholtz coils and its uniformity in the absence of magnetic materials are well known, e.g. $[6,8,9,20])$. Analysis of sensitivity of Helmholtz coils on imperfections of design parameters are conducted in a number of papers, see e.g. [2-4, 7, 17].

According to our best knowledge, this is the first consideration of the uniformity of magnetic field inside square Helmholtz coils enclosed with high-permeability material. In this subsection it is shown that the volume of uniform magnetic field may increase in the presence of the enclosure.

As an example we calculate magnetic flux density of square Helmholtz coils in the air (Fig. 5a), and with the enclosure by taking into account only the first layer of images (Fig. 5b). The magnetic flux density is calculated in the region $\Omega_{0}$ at the plane $y=0$. The values used in the calculations are $I_{0}=1 \mathrm{~A}$, $d=0.5 \mathrm{~m}, \quad a=0.2 \mathrm{~m}, \quad h=0.5445 a=0.1089 \mathrm{~m}$ and $\alpha=1$. It is easy to observe that the magnetic flux density around the center of the system is increased in the presence of the enclosure and at the same time, the volume of high uniformity of magnetic field is enlarged. In this example we also found that the effects of the second layer are weak and that the effects of the layers of order higher than two can be completely neglected.

The magnetic field non-uniformity $u$ within the system of the loops (expressed as a percentage) can be defined as [2]

$$
u[\%]=\left|1-B(x, y, z) / B_{0}\right| \cdot 100,
$$

where $B_{0}$ denotes the magnetic flux density at the center of the system (which for the coordinate system adopted in this paper coincides with the origin) and $B(x, y, z)$ denotes magnetic flux density at point $(x, y, z)$. Due to the symmetry of the system, magnetic flux density has only $z$-component on $z$-axis (axis of the system). According to the definition (5), the value $u=0$ corresponds to uniform field and low values of $u$ indicate low level of field non-uniformity. For most bio-magnetic experiments magnetic field non-uniformities of less than $10 \%$ or $5 \%$ are acceptable [15]. Very rare, non-uniformity of less than $1 \%$ is required. The lines in Fig. 6 represent the contours of 1\%, 2\%, 5\% and 10\% of non-uniformity of the magnetic field at the plane $y=0$.
Furthermore, we analyze non-uniformity of the magnetic field at the planes $z=0, z=0.1 \mathrm{~m}$, and $z=0.15 \mathrm{~m}$. Contour lines of $1 \%, 2 \%, 5 \%$ and $10 \%$ of non-uniformity are presented in Fig. 7 , Fig. 8 and Fig. 9, respectively. Dashed red lines indicate the positions of the square loops. Additionally, from the shape of the contours of constant non-uniformity $u$, it follows that $u$ can be considered as a function of $z$ and $r_{x y}=\sqrt{x^{2}+y^{2}}$.

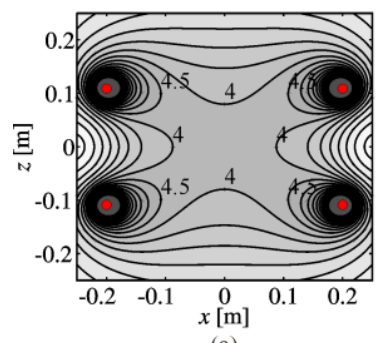

(a)

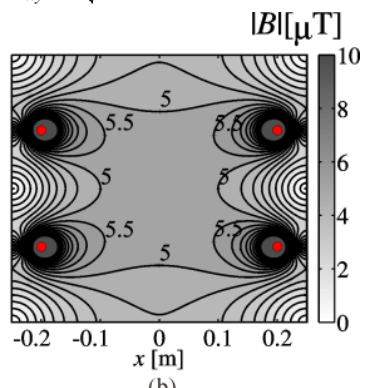

(b)
Fig. 5. Magnetic flux density of Helmholtz coils at the plane $y=0(a)$ without enclosure and (b) with enclosure by taking into account only the first layer of images
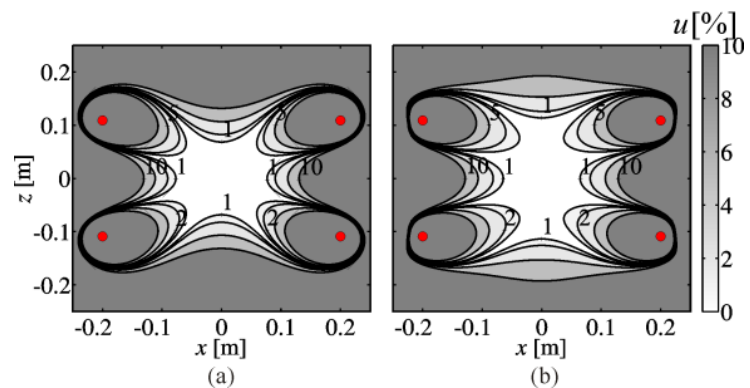

Fig. 6. Non-uniformity of the magnetic flux density of Helmholtz coils at the plane $y=O(a)$ without enclosure and $(b)$ with enclosure

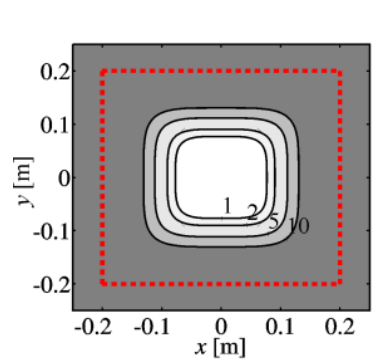

(a)

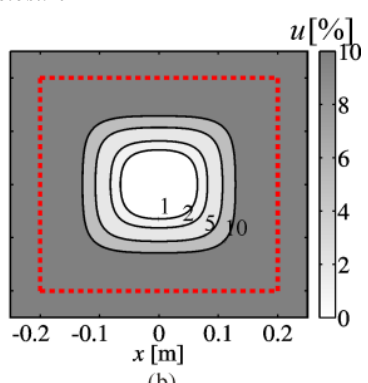

(b)
Fig. 7. Non-uniformity of magnetic flux density of Helmholtz coils at the plane $z=0$ (a) without enclosure and (b) with enclosure

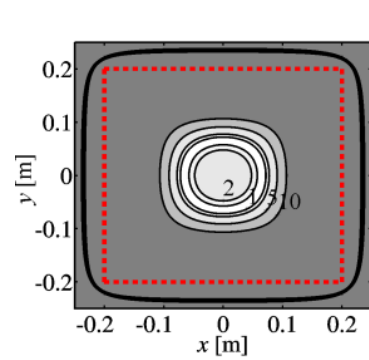

(a)

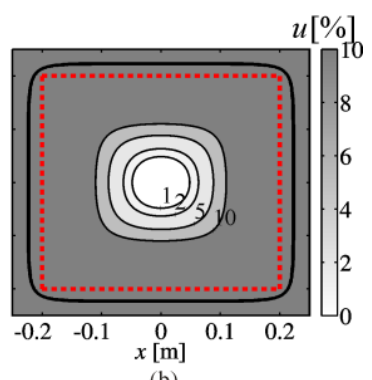

(b)
Fig. 8. Non-uniformity of magnetic flux density of Helmholtz coils at the plane $z=0.1 \mathrm{~m}(a)$ without enclosure and $(b)$ with enclosure

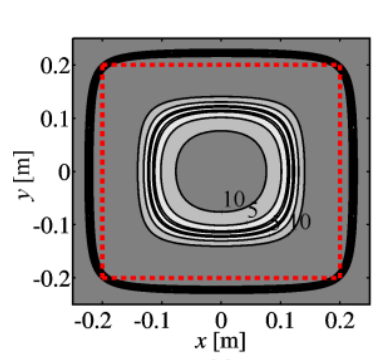

(a)

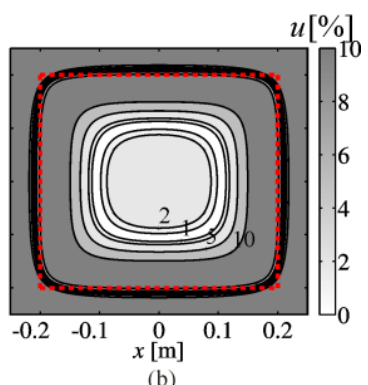

Fig. 9. Non-uniformity of magnetic flux density of Helmholtz coils at the plane $z=0.15 \mathrm{~m}(\mathrm{a})$ without enclosure and $(b)$ with enclosure 
Boundaries of the volume within $5 \%$ and $10 \%$ of nonuniformity inside Helmholtz coils with and without enclosure are summarized in Table 2. According to Table 2, the influence of the enclosure on lateral dimensions of the volume of uniform field is weak. On the other hand, the $z$-coordinates where $u \leq 5 \%$ is attained, are $|z / a| \leq 0.76$ and $|z / a| \leq 0.53$ for Helmholtz coils with and without enclosure, respectively. The $z$-coordinates where non-uniformity $u \leq 10 \%$ is attained are $|z / a| \leq 0.90$ and $|z / a| \leq 0.66$ for Helmholtz coils with and without enclosure, respectively. Consequently, the volume of the uniform field is significantly enlarged in the presence of the enclosure.

Table 2. Boundaries of volume of uniform field inside Helmholtz coils with and without enclosure

\begin{tabular}{|c|c|c|}
\hline$u$ & without enclosure & with enclosure \\
\hline \multirow{2}{*}{$5 \%$} & $r_{x y} / a \leq 0.45$ & $r_{x y} / a \leq 0.44$ \\
\cline { 2 - 3 } & $|z / a| \leq 0.53$ & $|z / a| \leq 0.76$ \\
\hline \multirow{2}{*}{$10 \%$} & $r_{x y} / a \leq 0.54$ & $r_{x y} / a \leq 0.55$ \\
\cline { 2 - 3 } & $|z / a| \leq 0.66$ & $|z / a| \leq 0.90$ \\
\hline
\end{tabular}

The aforementioned definition of non-uniformity (5) includes the magnetic flux density magnitude. However, the polarization of the uniform field should be linear, with the direction of the magnetic flux density vector along $z$-axis. In what follows, we consider two measures for non-uniformity of the $z$-component of magnetic flux density.

Let us first introduce relative difference between $z$-component of the magnetic flux density $B_{z}(0,0, z)$ at a point on the axis of the system and $B_{0}$ at the center of the system (that is, at the origin)

$$
\varepsilon[\%]=\left[1-B_{z}(0,0, z) / B_{0}\right] \cdot 100,
$$

as proposed in [23]. For Helmholtz coils, fraction $h / a$ is constant. Therefore, $\varepsilon$ depends only on $d / a$ and $z / a$ where $a$ denotes half-length of the sides of the loops and $d$ denotes length of the edges of the enclosure. From the geometry of the system, it immediately follows that $d / a>2$ and $d / a>2|z / a|$. Plots of the contours of absolute value of $\varepsilon$ as a function of $d / a$ and $|z / a|$ for Helmholtz coils without enclosure and with enclosure are presented in Figs. 10(a) and 10(b), respectively. The volumes with $|\varepsilon| \leq 5 \%$ and $|\varepsilon| \leq 10 \%$ are considerably increased in the presence of the enclosure for $d / a<3 \quad(d / a=2.5$ in the previous example presented in Figs. 6-9). For $d / a>3$, the enclosure is not close enough to the coils and its influence is weak. However, for $d / a<3$, the presence of the enclosure causes the increase of the height of the volume of the uniform field. This increase can be explained through the contribution of the four images in the first layer that are coaxial with the original loops and having the current in the same direction as in the original loops. Thus two original loops along with these four images form a system of six coaxial and parallel loops. Such virtual system may provide elongated volume of uniform magnetic field compared to the system consisting of the two coils alone.

Furthermore, let us introduce relative difference between $z$-component of the magnetic flux density $B_{z}(x, 0, z)$ at point $(x, 0, z)$ and $B_{0}$,

$$
\xi[\%]=\left[1-B_{z}(x, 0, z) / B_{0}\right] \cdot 100 .
$$

According to (7), $\xi$ depends on $d / a, x / a$, and $z / a$. Plots of the contour lines of absolute value of $\xi$ for Helmholtz coils with enclosure, $d / a \in[2,3]$ and the following six values of $|z / a|$, $|z / a| \in\{0,0.15,0.3,0.45,0.6,0.75\}$ are presented in Figs. 11(a)-(f), respectively. These figures confirm that high uniformity of the $z-$ component of the magnetic flux density can be also achieved in the presence of the enclosure.

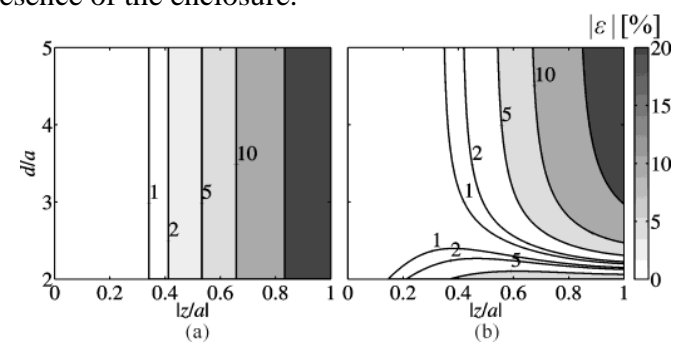

Fig. 10. Absolute values of relative difference $\varepsilon$ for Helmholtz coils (a) without enclosure and $(b)$ with enclosure

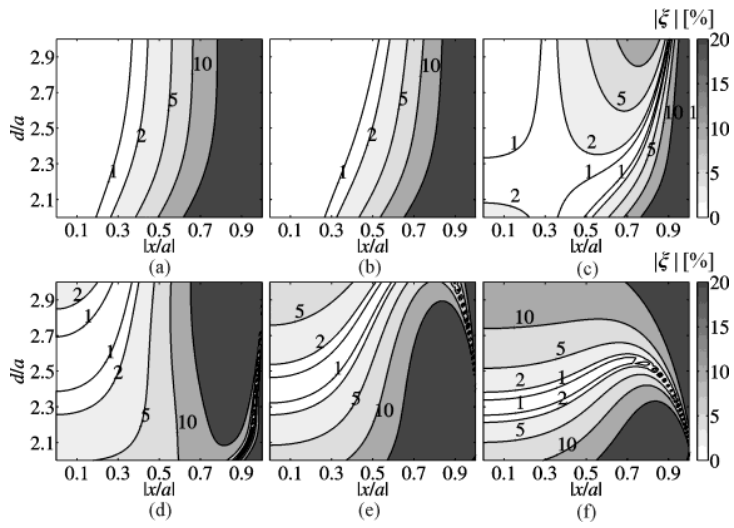

Fig. 11. Absolute values of relative difference $\xi$ for Helmholtz coils with enclosure for (a) $|z / a|=0$, (b) $|z / a|=0.15$, (c) $|z / a|=0.3$, (d) $|z / a|=0.45$, (e) $|z / a|=0.6$, (f) $|z / a|=0.75$

\section{Conclusion}

In this paper we analyze magnetic field of the square loop enclosed with a high-permeability material having infinitely thick walls. Closed form expressions for the positions and centers of an infinite set of the images are provided. The images are grouped into the subsets, so-called layers of images. The contribution of the layers to the magnetic field is investigated numerically. It is shown that the impact of the second layer is very weak, and the impact of the layers of the order higher than two can be completely neglected, providing that $\alpha \geq 0.5$ (corresponding to $\mu \geq 3 \mu_{0}$ ). In calculations of magnetic field only contributions of the images in the first layer should be included.

In Section 2 we consider the system of two coaxial and parallel identical square coils enclosed with high-permeability material with infinitely thick walls. Square Helmholtz coils, as an important special case, are considered in detail. We show that the volume of the uniform magnetic field inside the Helmholtz coils can be significantly increased in the presence of the enclosure. A possible application of these results is in the design of exposure system for in-vitro bio-magnetic experiments based on Helmholtz coils. Such system is usually placed inside the commercial incubator with heating elements, which produce magnetic field. Apart from shielding this field, the box made of high-permeability material can be used to enlarge the volume of uniform magnetic field inside the Helmholtz coils.

In this paper, we assume that the walls of the enclosure are infinitely thick, and made of material having constant permeability and zero conductivity. These conditions can be approximately fulfilled by using ferrite with relative permeability higher than 100 [18], providing that the saturation is avoided. Moreover, before the practical realization, the sensitivity of the magnetic field uniformity on the imperfections of the dimensions of the coils and their positions inside the box should be analyzed.

The presented work might be easily extended to include considerations of the systems of three or more coaxial and parallel square coils enclosed with high-permeability material. In future work we will also consider the enclosed systems of coaxial and parallel circular coils. 


\section{Appendix}

In this appendix we provide the expressions that enable fast and easy calculation of the magnetic flux density vector of the original and the image square loops.

A square loop in homogeneous medium of constant permeability $\mu$ is shown in Fig. 12. The side dimensions of the loop are $2 a$, center of the loop is at $\left(x_{0}, y_{0}, z_{0}\right)$, the plane of the loop is parallel to $x y$-plane and the edges of the loop are parallel to either $x$ or $y$ axis.

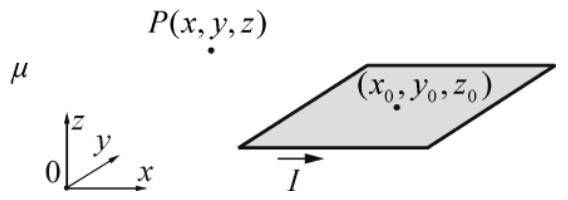

Fig. 12. Square loop

Following the derivation presented in [12], it is easy to show that the components of the magnetic flux density at point $P(x, y, z)$ can be expressed as

$$
\begin{gathered}
B_{x}(x, y, z)=\frac{\mu I\left(z-z_{0}\right)}{4 \pi} \sum_{k=1}^{4} \frac{(-1)^{k+1}}{r_{k}\left(r_{k}+d_{k}\right)}, \\
B_{y}(x, y, z)=\frac{\mu I\left(z-z_{0}\right)}{4 \pi} \sum_{k=1}^{4} \frac{(-1)^{k+1}}{r_{k}\left(r_{k}+c_{k}\right)}, \\
B_{z}(x, y, z)=\frac{\mu I}{4 \pi} \sum_{k=1}^{4}(-1)^{k}\left[\frac{c_{k}}{r_{k}\left(r_{k}+d_{k}\right)}+\frac{d_{k}}{r_{k}\left(r_{k}+c_{k}\right)}\right],
\end{gathered}
$$

where

$$
\begin{array}{ll}
c_{1}=\left(x-x_{0}\right)+a, & d_{1}=\left(y-y_{0}\right)+a, \\
c_{2}=\left(x-x_{0}\right)-a, & d_{2}=\left(y-y_{0}\right)+a, \\
c_{3}=\left(x-x_{0}\right)-a, & d_{3}=\left(y-y_{0}\right)-a, \\
c_{4}=\left(x-x_{0}\right)+a, & d_{4}=\left(y-y_{0}\right)-a,
\end{array}
$$

and

$$
r_{k}=\sqrt{c_{k}^{2}+d_{k}^{2}+\left(z-z_{0}\right)^{2}}, \quad k=1, \ldots, 4 .
$$

Magnetic flux density on the axis of the square coil can be obtained by substituting $x=x_{0}$ and $y=y_{0}$ into (8)-(12). After some rearrangements it follows that

$$
\begin{aligned}
& B_{x}\left(x_{0}, y_{0}, z\right)=0, \\
& B_{y}\left(x_{0}, y_{0}, z\right)=0, \\
& B_{z}\left(x_{0}, y_{0}, z\right)=\frac{2 \mu I a^{2}}{\pi\left[a^{2}+\left(z-z_{0}\right)^{2}\right] \sqrt{2 a^{2}+\left(z-z_{0}\right)^{2}}} .
\end{aligned}
$$

Finally, $z$-component of the magnetic flux density at the center of the loop is equal to

$$
B_{z}\left(x_{0}, y_{0}, z_{0}\right)=\frac{\sqrt{2} \mu I}{\pi a} .
$$

\section{Acknowledgement}

This work is supported by Serbian Ministry of Education, Science and Technology Development as a part of the Project TP32016.

\section{References}

[1] Ahuir J. V.: Going wireless with magnetic shielding. A on Note ANP016, Wurth Electronic Inc. 2013, http://www.we-online.com/web (available 12.12.2017).

[2] Beiranvand R.: Analyzing the uniformity of the generated magnetic field by a practical one-dimensional Helmholtz coils system. Rev. Sci. Instrum. 7/2013, 075109-1-11 [DOI: 10.1063/1.4813275].

[3] Beiranvand R.: Magnetic field uniformity of the practical tri-axial Helmholtz coils systems. Rev. Sci. Instrum. 5/2014, 055115-1-10 [DOI:
[DO 10.1063/1.4876480].

[4] Beiranvand R: Effects of the winding cross section shape on the magnetic field uniformity of the high field circular Helmholtz coil systems. IEEE Trans. Ind. Electron. 2017, 7120-7131 [DOI: 10.1109/TIE.2017.2686302].

[5] Bronaugh E. L.: Helmholtz coils for calibration of probes and sensors: Limits of magnetic field accuracy and uniformity. IEEE 1995 Int. Symp. Electromagn. Compatibility, Atlanta. 72-76 [DOI: 10.1109/ISEMC.1995.523521].
[6] Cacak R. K., Craig J. R.: Magnetic field uniformity around near Helmholtz coil configurations. Rev. Sci. Instrum. 11/1969, 1468-1470 [DOI:
[D. $10.1063 / 1.1683829]$

[7] Crosser M. S., Scott S., Clark A., Wilt P. M.: On the magnetic field near the center of Helmholtz coils. Rev. Sci. Instrum. 8/2010, 084701-1-6 [DOI: 10.1063/1.3474227]

[8] Crownfield F. K., Jr.: Optimum spacing of coil pairs. Rev. Sci. Instrum. 2/1964, 240-241 [DOI: 10.1063/1.1718796].

[9] Firester A. H.: Design of square Helmholtz coil systems. Rev. Sci. Instrum. 9/1966, 1264-1265 [DOI: 10.1063/1.1720478].

[10] Frix W. M., Karady G. G., Venetz B. A.: Comparison of calibration systems for magnetic field measurement equipment. IEEE Trans. Power Del. 1/1994, 100108 [DOI: $10.1109 / 61.277684]$.

[11] Hammond P.: Electric and magnetic images. Proceedings of the IEE - Part C: Monograph 12/1960, 306-313 [DOI: 10.1049/pi-c.1960.0047].

[12] Juhas A., Pekaric-Nad N., Toepfer H.: Magnetic field of rectangular current loop with sides parallel and perpendicular to the surface of high-permeability material. Serb. J. Electr. Eng. 4/2014, 701-717 [DOI: 10.2298/SJEE1404701J].

[13] Kalafala, A. K.: Optimized configurations for passively shielded magnetic resonance imaging magnets. IEEE Trans. Magn. 2/1993, 1240-1244 [DOI: $10.1109 / 20.250628]$

[14] Kirschvink J. L.: Uniform magnetic fields and double-wrapped coil systems: Improved techniques for the design of bioelectromagnetic experiments. Bioelectromagnetics 1992, 401-411 [DOI: 10.1002/bem.2250130507].

[15] Misakian M.: Exposure systems. EMF Engineering Review Symposium, Charleston, 1998. 4.1-4.6. ftp://ftp.emf-data.org/pub/emfdata/symposium98/topic-04-synopsis.pdf (available 12.12.2017).

[16] Poppenk F. M., Amini R., Brouwer G. F.: Design and application of a Helmholtz cage for testing nano-satellites. Proc. 58th Congress IAF, paper no. IAC-07-C1.8.02, Huderabad, India, 2007. 4650-4659.

[17] Purcell E. W.: Helmholtz coils revisited. Amer. J. Phys. 1989, 18-22 [DOI: 10.1119/1.15860].

[18] Roshen W. A.: Effect of finite thickness of magnetic substrate on planar inductors. IEEE Trans. Magn. 1/1990 270-275 [DOI: 10.1109/20.50553].

[19] Ruark A. E., Peters M. F.: Helmholtz coils for producing uniform magnetic fields. J. Opt. Soc. Am. 1926, 205-212 [DOI: 10.1364/JOSA.13.000205].

[20] Rudd M. E., Craig J. R.: Optimum spacing of square and circular coil pairs. Rev. Sci. Instrum. 9/1968, 1372-1374 [DOI: 10.1063/1.1683678]

[21] Schuderer J., Oesch W., Felber N., Spat D., Kuster N.: In vitro exposure apparatus for ELF magnetic fields. Bioelectromagnetics 2004, 582-591 [DOI: 10.1002/bem.20037].

[22] Turowski J., Turowski M.: Engineering Electrodynamics Electric Machine, Transformer, and Power Equipment Design, CRC Press Taylor \& Francis Group 2014

[23] Wang J., She S., Zhang S.: An improved Helmholtz coil and analysis of its magnetic field homogeneity. Rev. Sci. Instrum. 5/2002, 2175-2179 [DOI $10.1063 / 1.1471352]$

\section{Ph.D. Anamarija Juhas}

e-mail: ajuhas@uns.ac.rs

Anamarija Juhas received Ph.D degree in electrical engineering from the Faculty of Technical Sciences (FTN), University of Novi Sad, in 2009. She is currently an Associate Professor with the Department of Power, Electronic and Telecommunication Engineering at the same Faculty. Her research interests include calculation and measurements of electric and magnetic fields, electromagnetic compatibility, human exposure to electromagnetic fields, memristors and power amplifiers.

\section{Prof. Neda Pekaric Nad}

e-mail: pecaric@uns.ac.r

Neda Pekaric Nad got her Ph.D degree in Electrical engineering from the Faculty of Electrical Engineering (ETF), University of Belgrade, in 1984. For many years she is a Professor at the Department of Power, Electronic and Telecommunication Engineering at the University of Novi Sad. Her research interests include electromagnetic compatibility, as well as study of electric and magnetic fields and their biological effects.

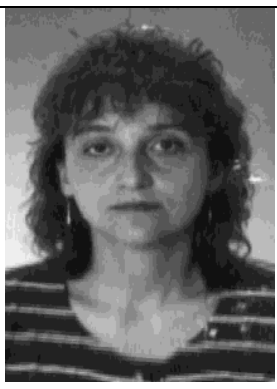

\section{Prof. Hannes H. Toepfer}

e-mail: hannes.toepfer@tu-ilmenau.de

Hannes Toepfer studied Electrical Engineering at the Institute of Technology Ilmenau. From 1991-2002 he worked as an scientific assistant at the Ilmenau University of Technology. In 1996, he received the Dr.-Ing. (equiv. $\mathrm{PhD}$ ) and in 2003 his postdoctoral qualification (Habilitation). In 2002, he joined the Institute for Microelectronics and Mechatronics Systems where he has been directing the System Design department. In 2009, he became a Full Professor at Technische Universität Ilmenau and heading the Advanced Electromagnetics Group with a research focus on electromagnetic sensors. 